\title{
DETECTION AND LOCALIZATION OF TRAFFIC LIGHTS USING YOLOV3 AND STEREO VISION
}

\author{
Wael Omar ${ }^{1 *}$, Impyeong Lee ${ }^{1}$, Gyuseok Lee ${ }^{1}$, Kang Min Park ${ }^{1}$ \\ ${ }^{1}$ Dept. of Geoinformatics, University of Seoul, Seoul, Republic of Korea - (uosgrad2018009, iplee, ys96000,)@uos.ac.kr, \\ minpkang@gmail.com
}

Commission II, WG II/ 1

KEY WORDS: traffic lights, detection, localization, convolutional neural network, stereo vision.

\begin{abstract}
:
This paper focus on traffic light distance measurement using stereo camera which is a very important and challenging task in image processing domain, where it is used in several systems such as Driving Safety Support Systems (DSSS), autonomous driving and traffic mobility. In this paper, we propose an integrated traffic light distance measurement system for self-driving based on stereo image processing. Therefore, an algorithm to spatially locate the detected traffic light is required in order to make these detections useful. In this paper, an algorithm to detect, classify the traffic light colours and spatially locate traffic light are integrated. Detection and colours classification are made simultaneously via YOLOv3, using RGB images. 3D traffic light localization is achieved by estimating the distance from the vehicle to the traffic light, by looking at detector 2D bounding boxes and the disparity map generated by stereo camera. Moreover, Gaussian YOLOv3 weights based on KITTI and Berkeley datasets has been replaced with the COCO dataset. Therefore, a detection algorithm that can cope with mislocalizations is required in autonomous driving applications. This paper proposes an integrated method for improving the detection accuracy and traffic lights colours classification while supporting a realtime operation by modelling the bounding box (bbox) of YOLOv3. The obtained results show fair results within 20 meters away from the sensor, while misdetection and classification appeared in further distance.
\end{abstract}

\section{INTRODUCTION}

Our transportation system must be autonomous to avoid accident scenarios. The vehicles should have eyes, not real eyes, but cameras for these things to happen. Traffic lights are also one of these important objects. Since the drivers are often wrong in complying with the rules of traffic light, traffic lights detection is considered to be very important because they are a part of public safety.

A variety of algorithms have been used for traffic signs and lights as well. Ultimately, integrating different methods, detection, color recognition and distance calculation, into one system is essential for an autonomous vehicle for safety reason. Safety is not only for drivers and passengers but also

for pedestrians, other vehicles and two-wheelers. In order to be widely accepted, safety issues must be resolved to the full satisfaction of the people. Self-driving vehicles are now part of our transport network. The fast development of automotive technology focuses on giving us the best safety features and Automated Driving Systems (ADS) in vehicles can handle the entire work of driving when the person wants the vehicle to switch to an auto-driving mode or when the person is unsure of driving. Self-driving vehicles and trucks that drive us will become a reality instead of us driving them. Object detection is necessary to achieve all these things.

Object detection is now commonly used as a major software system in self-driving cars for the detection of objects such as pedestrians, cars, traffic lights etc. The scenario is even worse in cases of drunk driving, where the driver would lose control and hit other vehicles and will not stop at the traffic lights, leading to major accidents and even death.

De Charette et al (Raoul de Charette, 2009)suggested a threestep procedure. spotlight detection is executed in the grey level image by using top hat morphological operator to highlight high-intensity spots.

$\mathrm{Mu}$ et al (G. Mu, 2015)proposed an image processing approach that converts the image color from red green blue (RGB) to hue-saturation value (HSV). Potential areas were then identified by scanning the scene using transcendental colour threshold with prior knowledge of the image. Finally, it was identified the location of the traffic lights using the Oriented Gradients (HOG) and Support Vector (SVM) functionalities. But before doing the traffic light classification YOLOv3 predicts an object score for each bounding box. Therefore, a detection algorithm that can cope with mislocalizations is required in autonomous driving applications. This algorithm was applied for improving the detection accuracy while supporting a real-time operation of YOLOv3, which is the most representative of one-stage detectors, with a Gaussian parameter and redesigning the loss function (Lee J. C.-J., 2019).

\section{RELATED WORK}

Traffic lights usually have startling colours so drivers can easily see them. These colours make them easily detectable using color filters (Fleyeh, 2004), however, these approaches require manual tuning of the thresholds for colour filtering, resulting in a difficult task because illumination and weather

\footnotetext{
* Corresponding author
} 
conditions will affect the colors. Furthermore, traffic lights have different orientation in horizontal or vertical.

Much research is taking place in the field of autonomous vehicle manufacturing, such as the detection by these vehicles of traffic lights and road signs. These vary with the techniques used, with regard to the environment and the cars to be used. Ozcelik et al proposed A Vision Based Traffic Light Detection and Intelligent Vehicle Recognition Approach (Ozcelik, 2017). Images are taken using a camera, and processing to detect the traffic is performed stepwise. The color of the traffic light is easily identified through the classification model Support Vector Machines (SVM), which is a machine learning algorithm prepared beforehand, after the location of the traffic lights is determined in the image. Muller et al have proposed Detecting Traffic Lights through Single Shot Detection technique which performs object proposals creation and classification using a single CNN (Müller, 2018).

A deep learning approach was proposed to Traffic Lights by detection, tracking, and classification (Behrendt, 2017). This proposed methodology provides a stable system consisting of a detector, tracker, and classifier depending on deep learning, stereo vision, and vehicle odometry that considers traffic lights in real-time.

Li et al proposed a "Traffic Light Recognition Technique" for the Complex Scene with Fusion Detections (Li, 2018). Saini et al proposed a Vision-Based Traffic Light Detection and State Recognition Technique for Autonomous Vehicles (Saini, 2017). It provides a vision-based technique for detecting traffic light structure using $\mathrm{CNN}$ that is based on a state recognition method that is considered to be reliable under different illumination and weather conditions. Shi et al. proposed the Adaptive Background Suppression Filter RealTime Traffic Light Detection (Shi, 2016).

Hamdi et al have proposed an ANN Real-Time Implementation classification system for road signs (Hamdi, 2017). This system provides a real-time algorithm to classify traffic signs by way of a driver alert system and recognize them. A traffic sign recognition system using hybrid descriptor features and an artificial neural network classifier has been suggested by Abedin et el (Abedin, 2016).

Many methodologies for traffic light detection are presented in the research works mentioned here. But due to the presence of different drawbacks these methodologies are also hampered. The systems presented fail to consider a wide variety of data sets for both training and testing, which can be considered to scale the systems accuracy.

\section{PROPOSED INTEGRATED SYSTEM}

The proposed integrated system aims to detect, localize, and measure the distance between the camera and traffic light while performing real time traffic lights recognition. This can be achieved by implementing machine learning and image classification techniques. Artificial Neural Networks, Convolution Neural Networks and in specific, are one of the most accurate methods in order to achieve the desired result.

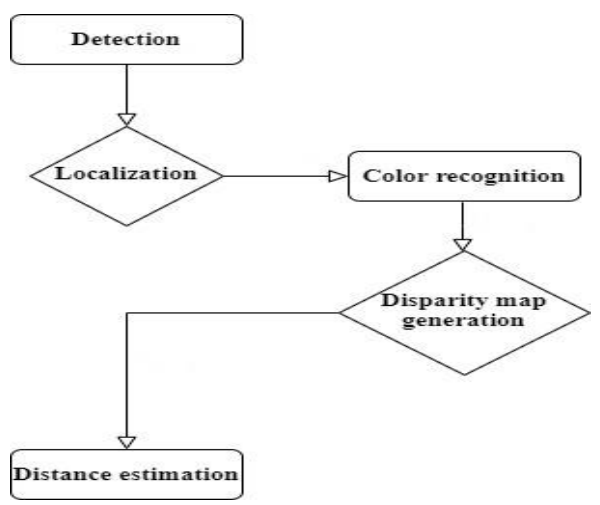

Figure 1. shows the flow of integrated system

CNNs have recently become popular because of their speed and precision in detecting objects. A popular CNN object detector is Faster R-CNN, which consists of two CNNs: the first one proposes input image regions of interest, and the second one refines and classifies those regions. In (Zuo, 2017), a plain Faster R-CNN was used to detect traffic signs in this research, but the detector struggled because signs are commonly a small part of the image, making the detection task more difficult.

A modified Faster R-CNN was developed in (Wang, 2018), resulting in a more efficient process for detecting signs of traffic signs. Although it has a great performance, having two detection and classification stages makes the processing slower if it was one stage.

One stage detectors are faster because they propose regions and at the same time classify them as high-speed detectors, such as OverFeat (Sermanet, 2013), SSD (Liu, et al., 2016) and YOLO (J. Redmon, 2016), (Redmon, 2016), (Redmon, 2018). A simultaneous SSD-based traffic signal detection and classification method is presented in (Lee H. S., 2018), which results in high accuracy; however, its main drawback is that each image with a resolution of $533 \times 300$ is processed in 0.126 seconds.

The modified YOLOv2 achieved 0,017 seconds per image $608 \times 608$ with a processing rate of 0,017 seconds, maintaining a high level of precision in the detection of traffic signs ( $\mathrm{J}$. Zhang, 2017). This shows that YOLOv2 can be used in the detection of traffic lights as well and that YOLOv3 has improved its function extractor and network architecture (lowering its processing speed), thus providing traffic lights detention tasks using YOLOv3 better accuracy but rather slower results.

\subsection{Detection}

YOLOv3 is a real-time detector and classifier based on CNN that has great performance in detecting small objects and is a perfect choice for this task due to the usual size of the traffic lights. But because YOLOv3 uses the sum of the squared error loss for bbox, it results with noisy data. However, utilize new model that cope with the loss function of bbox makes the model more robust against the noisy environment. Therefore, Gaussian YOLOv3 can obtain the uncertainty of bbox for every detection object in an image (Lee J. C.-J., 2019). By utilizing Gaussian YOLOv3, an improvement in the mean average precision (mAP) by 3.09 and 3.5 on the KITTI and 
Berkeley deep drive (BDD) datasets, respectively. This pretrained model has been selected as it is capable of real-time detection at faster than 42 frames per second (fps) and shows a higher accuracy than previous approaches with a similar fps. Therefore, the proposed algorithm is the most suitable pretrained model for autonomous driving applications and localize traffic light position (Jiwoong Choi, 2019).

The performance of the selected pretrained model using the KITTI validation set compared to YOLOv3, the Gaussian YOLOv3 mAP improves by 3.09 , with a detection speed of $43.13 \mathrm{fps}$, allowing real-time detection with a slight variation from YOLOv3. Gaussian YOLOv3 is $3.93 \mathrm{fps}$ faster than RFBNet (Songtao Liu, 2018), which has the fastest operating speed in previous studies with the exception of YOLOv3, although Gaussian YOLOv3's mAP exceeds RFBNet (Songtao Liu, 2018) by more than 10.17 .

\subsection{Localization}

The 2D position of the traffic lights acquired during the detection phase does not provide enough information for an autonomous vehicle to make a decision. A method for obtaining their depth is required to locate traffic light correctly, which means that the decision-making algorithm receives more information and therefore improves autonomous driving. Although CNNs have been included with the neural detection network for the estimation of the monocular camera depth (I. Laina, 2016), (L. He, 2018), (D. Eigen, 2014), it will slow the entire procedure; because computer resources are distributed. Therefore, the optimal solution is an end-to-end CNN which detects and predicts at the same time the distance from the vehicle; however, this cannot be trained because no data set with traffic light boundary boxes and their distance is available.

On the other hand, with low computational resources, stereo vision approaches are fast enough to post-process the detections in real-time. Moreover, the calculation of depth with small errors is precise enough. Figure 2(a), shows an example of one stereo camera frame.
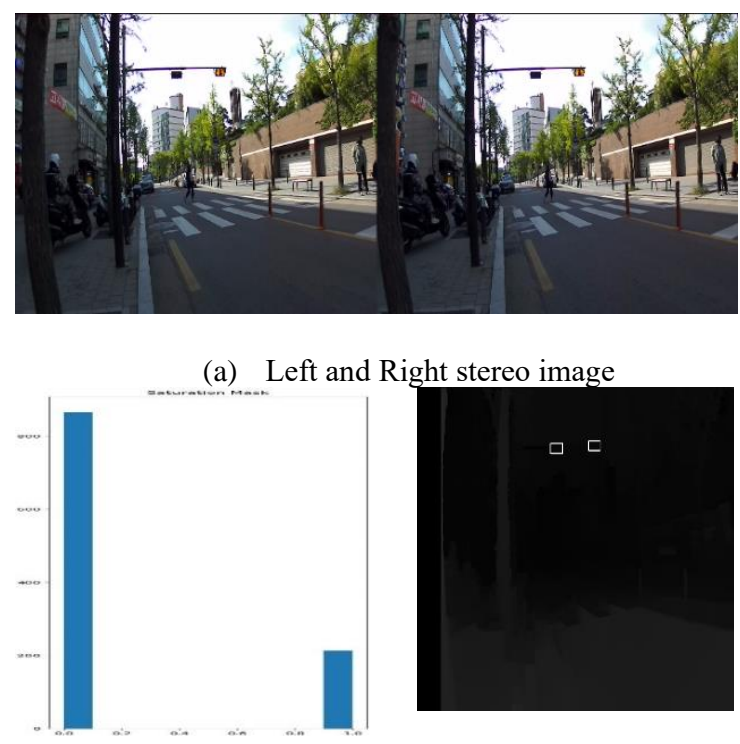

(b) Saturation mask

(c) Disparity map with the detected bounding boxes

\subsection{Traffic Light Recognition}

The recognition of traffic light and its colours can be divided into two steps. Firstly, locate the traffic light accurately and cut out the area of interest (ROI) around the location to reduce the calculation, and then through the image processing to achieve the final identification of traffic light (Xiaoqiang Wang, 2018).In this part an effective traffic light identification and colour classification scheme based on TensorFlow API and HSV color space was integrated.

Hence, to track the precise bounding box of the traffic light in the image. The obtained corner information is recorded for cropping region of interest (ROI) image from the original input. OpenCV is then used to smooth the ROI image and improve contrast. After converting the ROI image from BGR to HSV colour space, the result of the traffic light can be consulted on the $\mathrm{H}$ channel according to the area of the connected domain (Xiaoqiang Wang, 2018). The ROI image is transferred from the BGR space to the HSV colour space, and then the $\mathrm{H}$ (hue) component is separated therefrom to make a traffic light determination (Su X, 2017), (G, 2014) as shown in Figure 2.

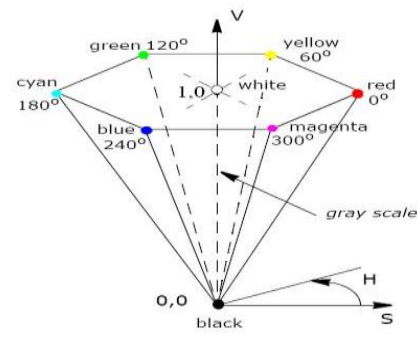

Figure 2. HSV colour model.

Then after detecting a traffic light we use the HSV method to classify the colour, isolating the saturation channel and visualizing the distribution. Figure $3(a, b$, and $c)$, we find that saturation is high at most of the area as the traffic light body is very good shaped. Choosing the area with the high saturation and high hue values as the area to mask yielded a good result as expected as shown in Figure3 (d, e, and f). Then plotting the hue image at the area of the light, it's obvious that's green is the highest ratio of them all as shown in Figure 3 (g, h, and i).

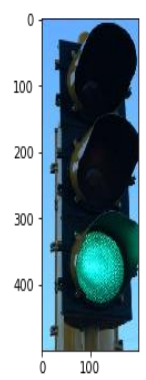

(a) Original

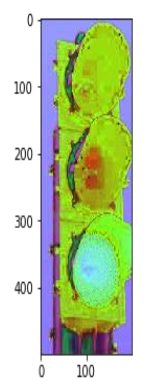

(b) Saturation

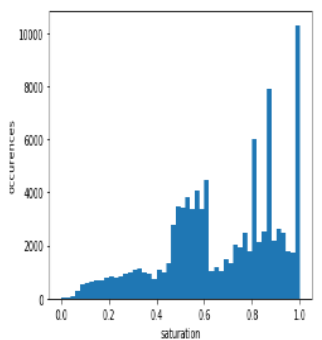

(c) Saturation Plotting

Fig. 2. Stereo images and their corresponding disparity map 


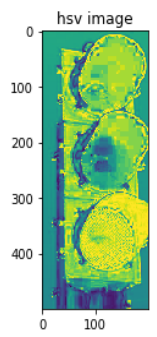

(d) Hsv

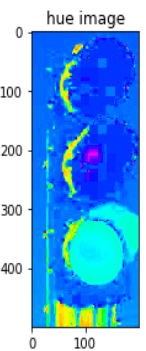

(g) Hue

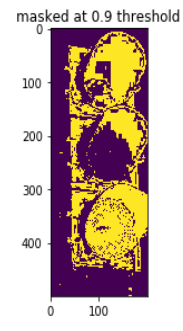

(e) 0.9

threshold

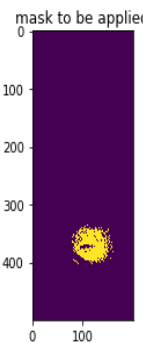

(h) Masked

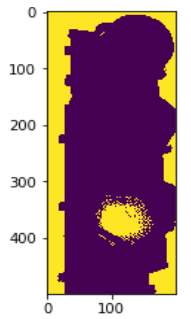

(f) Result

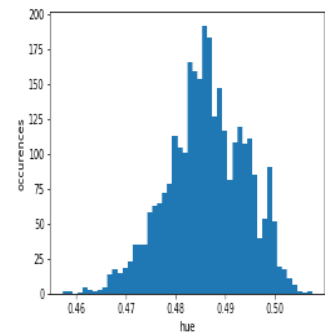

(i) Hue plotting
Fig. 3. HSV classification method

Finally, we extract the $\mathrm{H}$ channel and the results can be obtained by calculating the connected domain area and setting the threshold on the H channel as shown in Figure 4.

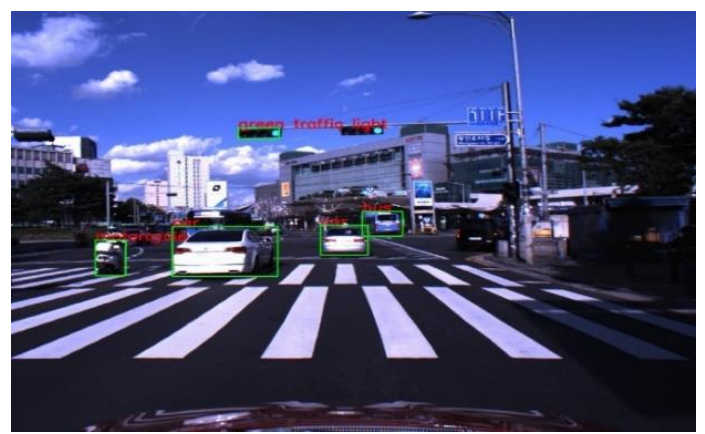

Figure 4. Results of traffic light detection and colour classification

\subsection{Disparity Map Generation}

Recreation of a three-dimensional representation of our images, we need to estimate the distance of every point. In the scene (which corresponds to a pixel in the image) of our cameras. The first thing we need is a disparity map. To calculate this, we initially implemented a simple block matching algorithm, using the Sum of Absolute Distances (SAD) metrics to match each pixel of the image captured from our right camera to a pixel in the image captured from our left camera.

The idea behind the algorithm is to find how much each pixel has shifted horizontally from one image to the other and from this information triangulate the corresponding point in space. The amount of shift is inversely proportional to the pixel's distance from the cameras: objects closer to the cameras will shift more from one image to the other, while our infinity point won't move at all.
The precision to which we can estimate the disparity value of a pixel (and consequently its depth in the scene) is limited by the pixel density of our image. To go beyond this limitation that restricts our disparity to scalar values, we can apply "Subpixel Estimation". Once we have identified our best match with the basic block matching algorithm, we take the corresponding minimum SAD and the SAD values of the neighbouring pixels and estimate a quadratic curve connecting the three. We then compute the minimum of this function by zeroing the derivative and this will be our new disparity value. Applying Subpixel Estimation yields a slightly smoother depth transition which especially enhances flat surfaces.

The stereo images are processed by looking at the position difference of each pixel in both images, generating a disparity map as shown in Figure 2(c). This disparity map shows the apparent motion of each pixel between the two stereo images. A higher motion indicates a pixel near, and a lower motion indicates a further pixel. Equation 1 is used for depth calculation of each pixel of two parallel cameras in metric units.

$$
Z=\frac{f T}{d}
$$

Where $\mathrm{Z}$ represents the depth of the selected pixel in the metric units. $f$ is the focal length, i.e. the distance from the focal point to the optical center of the lens. $T$ is the baseline, that is, the distance between the two cameras. Both $\mathrm{f}$ and $\mathrm{T}$ are obtained by the process of camera calibration. The camera used for this experiment is the ZED stereo camera; The images have a resolution of $2 \times(1280 \times 720$ pixels resolution), 60fps and have the following characteristics: focal length $\mathrm{f} 2.8 \mathrm{~mm}\left(0.11^{\prime \prime}\right)$ $\mathrm{f} / 2.0$, baseline $\mathrm{T}$ of $120 \mathrm{~mm}$ (4.7"), and pixel size $2 \mu \mathrm{m}$.

Combining equation (1) and the detected bounding boxes, the distance from each traffic light to the camera in the image can be estimated as follows: First, the 2D coordinates of each bounding box are projected in the disparity map of the Region of Interest (ROI) as shown in Figure 2(c). Then equation (1) is applied to each pixel inside that ROI and, finally, a histogram with distance intervals of 0.1 meters versus the number of pixels representing each distance is constructed. The distance for each bounding box is the most repeated value in the histogram. The histogram containing the distance values within the prediction bounding box is shown in Figure 5 . Histograms of further traffic lights show that the most frequent value is less dominant than the histograms of closer traffic lights.

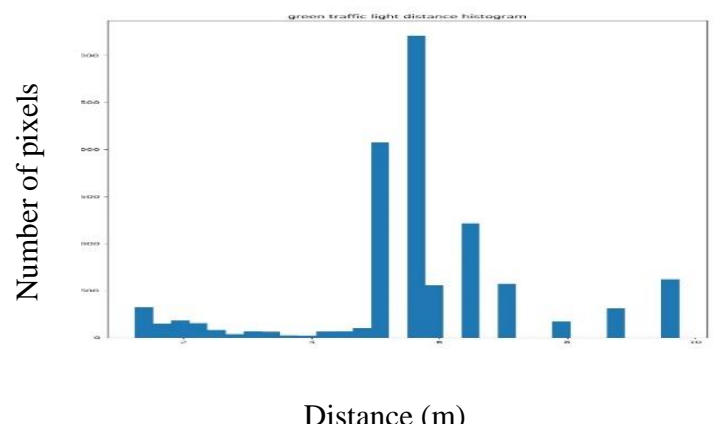

Fig. 5. Histogram of pixels inside the traffic light bounding box 


\section{EXPERIMENTAL RESULTS}

\subsection{Experiment Platform}

The platform is a portable tripod and is equipped with a front stereo camera described in section 3.4. The processing unit is equipped with an AMD Ryzen 73700X8-Core Processor and NVIDIA GeForce RTX 2060.

\subsection{Results and Evaluation}

3D Localization: Table 1 shows the real coordinates of each camera positions and the traffic light as shown in Figure1 (a) for different distance ranges.

\begin{tabular}{|l|c|c|c|}
\hline Positions & $\mathrm{X}(\mathrm{N})$ & $\mathrm{Y}(\mathrm{E})$ & $\mathrm{Z}(\mathrm{H})$ \\
\hline Traffic light & 553771.850 & 204838.802 & 41.212 \\
\hline Camera position1 & 553761.480 & 204904.980 & 36.942 \\
\hline Camera position2 & 553757.984 & 204880.639 & 36.854 \\
\hline Camera position3 & 553764.804 & 204860.660 & 36.603 \\
\hline Camera position4 & 553766.846 & 204850.749 & 36.528 \\
\hline
\end{tabular}

Table 1. Different 4 positions on different distance were measured away from one traffic light

To calculate the distance, enter two sets of coordinates in the three-dimensional Cartesian coordinate system, (X1, Y1, Z1) and (X2, Y2, Z2), to get the distance formula calculation for the 2 points and calculate distance between the 2 points. The distance between two points is the length of the path connecting them. The shortest path distance is a straight line.

In a 3-dimensional plane, the distance between points (X1, $\mathrm{Y} 1, \mathrm{Z} 1)$ and $(\mathrm{X} 2, \mathrm{Y} 2, \mathrm{Z} 2)$ is given by distance of each traffic sign was measured using the Total Station; Tie Distances calculates the distance and height differences between our stereo camera and the traffic light. This missing line measurement represents the real distance between our stereo camera as a centre point and multiple existed traffic light. This radial function can accurately calculate the distances to points P1 and Px, and the total station calculates both distance $\mathrm{d}$ and height difference $\mathrm{H}$ as shown in Figure 6.

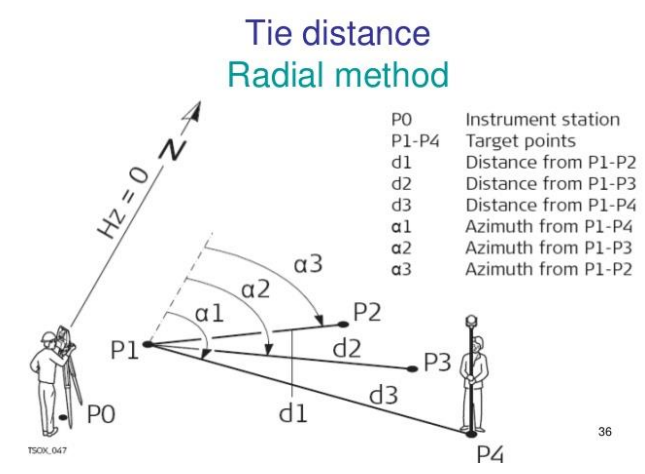

Figure 6. shows how to calculate different distance in different position

\begin{tabular}{|l|c|c|}
\hline Positions & $\begin{array}{c}\text { Measured } \\
\text { distance }(\mathrm{m})\end{array}$ & $\begin{array}{c}\text { Estimated } \\
\text { distance(m) }\end{array}$ \\
\hline Camera position 1 & 67.132 & 0.0 \\
\hline Camera position 2 & 44.289 & 0.0 \\
\hline Camera position 3 & 24.359 & 21.518 \\
\hline Camera position 4 & 13.773 & 10.845 \\
\hline
\end{tabular}

Table 2. shows 4 positions on different distance away from one traffic light

\section{CONCLUSION}

The Gaussian YOLO network is good for real time execution, but the Map isn't very good compared to Faster RCNN which appeared during the 2D detection part. The Zed camera faces accuracy problems when measuring points depth greater than 3 metres. The Zed camera didn't pick up certain features in the outdoor experiment that the monocular cameras can see particularly in locations where piles of material are positioned with shadows. Under different lighting conditions we captured the scene at varying speeds, framerates and resolution without any significant changes.

Using HSV yields a good accuracy but could not recognize and classify the colour of traffic lights from a far distance. Moreover, the disparity map for depth prediction is good for urban environments, but it is not very efficient in a very wide environment where you want to get the depth of a very distant object.

If real time execution won't be a problem replacing Gaussian YOLOV3 with Faster RCNN or SSD will be better. Trying to Add traffic light color classification to our Network by combining multiple datasets. Using sensor fusion, unscented Kalman Filter (UKF) by fusing the predicted depth for an object from the stereo camera disparity map and the lidar readings. Adding the Two-point clouds of both the camera and the LiDAR together and predicting the depth from the corrected output.

\section{ACKNOWLEDGMENT}

This research supported by Basic Research Program through the National Research Foundation of Korea(NRF) funded by the Ministry of Science, ICT \& Future Planning (NRF-2017R1A2B412908).

\section{REFERENCES}

Abedin, M. Z. (2016). Traffic sign recognition using hybrid features descriptor and artificial neural. IEEE.19th International Conference on Computer and Information Technology (ICCIT).

Behrendt, K. L. (2017). A deep learning approach to traffic lights: Detection, tracking, and classification. IEEE International Conference on Robotics and Automation (ICRA).

D. Eigen, C. P. (2014). Depth map prediction from a single image using a multi-scale deep network. 2366-2374. 
Fleyeh, H. (2004). Color detection and segmentation for road and traffic signs. Cybernetics and Intelligent Systems, 2004 IEEE Conference, 809-814.

Fleyeh, H. (2005). Road and traffic sign color detection and segmentation a fuzzy approach. 124-127.

G, R. H. (2014). Automatic Tracking of Traffic Signs Based on HSV C. International Journal of Engineering Research and Technology.

G. Mu, Z. X. (2015). Traffic light detection and recognition for autonomous vehicles. The Journal of China Universities of Posts and Telecommunications, 50-56.

Hamdi, S. e. (2017). Road signs classification by ANN for real-time implementation. IEEE International Conference on Control, Automation and Diagnosis (ICCAD). .

I. Laina, C. R. (2016). Deeper depth prediction with fully convolutional residual networks in 3D Vision (3DV). Fourth International Conference IEEE, 239-248.

J. Redmon, S. D. (2016). "You only look once: Unified, realtime object detection,". IEEE conference on computer vision and pattern recognition, 779-788.

J. Zhang, M. H. (2017). "A real-time chinese traffic sign . detection algorithm based on modified yolov2,", vol. 10, no. 4, p. 127 ,

Jiwoong Choi, D. C.-J. (2019). Gaussian YOLOv3: An Accurate and Fast Object Detector Using Localization Uncertainty for Autonomous Driving. The IEEE International Conference on Computer Vision, 502-511.

L. He, G. W. (2018). Learning depth from single images with deep neural network embedding focal length. IEEE Transactions on Image Processing.

Lee, H. S. (2018). "Simultaneous traffic sign detection and boundary estimation using convolutional neural network,. IEEE Transactions on Intelligent Transportation Systems.

Lee, J. C.-J. (2019). Gaussian YOLOv3: An Accurate and Fast Object Detector Using Localization Uncertainty for Autonomous Driving. IEEE.

Li, X. e. (2018). Traffic light recognition for complex scene with fusion detections. IEEE Transactions on Intelligent Transportation Systems .

Liu, W., Anguelov, D., Erhan, D., Szegedy, C., Reed, S., Fu, C.-Y., \& Berg, A. C. (2016). SSD: Single Shot MultiBox Detector. Lecture Notes in Computer Science, 21-37.

Müller, J. a. (2018). Detecting traffic lights by single shot detection. 21st International Conference on Intelligent Transportation Systems (ITSC). IEEE.

Ozcelik, C. T. (2017). A vision-based traffic light detection and recognition approach for intelligent vehicles. International Conference on Computer Science and Engineering (UBMK). IEEE.

Raoul de Charette, F. N. (2009). Traffic light recognition using image processing compared to learning processes.
IEEE/RSJ International Conference on Intelligent Robots and Systems, 333-338.

Redmon, J. F. (2016). Yolo9000: Better, faster, stronger. arXiv.

Redmon, J. F. (2018). "Yolov3: An incremental improvement,". arXiv.

Saini, S. e. (2017). An efficient vision-based traffic light detection and state recognition for autonomous vehicles. IEEE Intelligent Vehicles Symposium (IV).

Sermanet, P. D. (2013). Overfeat: Integrated recognition, localization and detection using convolutional networks. arXiv preprint.

Shi, Z. Z. (2016). Real-time traffic light detection with adaptive background suppression filter. IEEE Transactions on Intelligent Transportation Systems , 690-700.

Songtao Liu, D. H. (2018). Receptive field block net for accurate and fast object detection. In Proceedings of the European Conference on Computer Vision (ECCV), 385400.

Su X, C. X. (2017). HSV color space and adaptive shape support window based local stereo matching algorithm. J. Laser \& Optoelectronics, 1-13.

T.-Y. Lin, P. D. (2017). "Feature pyramid networks for object detection.,". CVPR, vol. 1, p. 4,

Wang, J. L. (2018). Real-time traffic sign recognition based on efficient cnns in the wild. IEEE Transactions on Intelligent Transportation Systems, 1-10.

Wei Liu, D. A.-Y. (2016). Ssd: Single shot multibox detector. in European conference on computer vision,, 21-37.

Xiaoqiang Wang, X. C. (2018). Design of traffic light identification scheme based on TensorFlow and HSV color space. Journal of Physics.

Z. Zuo, K. Y. ( 2017). Traffic Signs Detection Based on Faster R-CNN. IEEE 37th International Conference on Distributed Computing Systems Workshops (ICDCSW), Atlanta, 286-288.

Zuo, Z. Y. (2017). Traffic signs detection based on faster rcnn. IEEE 37th International Conference on Distributed Computing Systems Workshops (ICDCSW), Atlanta, GA, 286-288. 